\title{
A potential peptide vector that allows targeted delivery of a desired fusion protein into the human breast cancer cell line MDA-MB-231
}

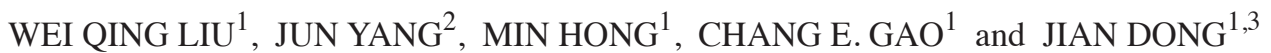 \\ Departments of ${ }^{1}$ Internal Medicine Oncology and ${ }^{2}$ Oncology, First Affiliated Hospital of Kunming Medical University, \\ Kunming, Yunnan 650032; ${ }^{3}$ Department of Internal Medicine Oncology, \\ Third Affiliated Hospital of Kunming Medical University, Kunming, \\ Yunnan 650106, P.R. China
}

Received December 24, 2014; Accepted March 8, 2016

DOI: $10.3892 / \mathrm{ol} .2016 .4538$

\begin{abstract}
Effective control of breast cancer has been primarily hampered by a lack of tumor specificity in treatments. One potential way to improve targeting specificity is to develop novel vectors that specifically bind to and are internalized by tumor cells. Through a phage display library, an 11-L-amino acid peptide, PI (sequence, CASPSGALRSC), was selected. PI was labeled with fluorescein isothiocyanate (FITC) and named PI-FITC. Subsequently, the specific affinity of PI-FITC to MDA-MB-231 human breast cancer cells and other cancer cell lines was observed by confocal microscopy. Our previous study established that PI-FITC also shows affinity to Calu-1 human lung carcinoma cells and major histocompatibility complex class I antigen molecules; therefore, the cytomembrane proteins of the cell lines were analyzed to determine those that were common to the two cell lines and may be associated with transmembrane transduction. To further test the delivery ability of PI to MDA-MB-231 cells, PI-glutathione-S-transferase (GST) was constructed and the internalization of this fusion protein was visualized by immunofluorescence microscopy. The results revealed that PI exhibited specific affinity to MDA-MB-231 cells. Use of membrane transport inhibitors indicated that macropinocytosis and caveolin-mediated endocytosis may be involved in the endocytosis of PI. In addition, 11 membrane proteins common to MDA-MB-231 and Calu-1 may be associated with transmembrane transduction. In summary, PI was able to deliver
\end{abstract}

Correspondence to: Dr Jian Dong, Department of Internal Medicine Oncology, Third Affiliated Hospital of Kunming Medical University, 519 Kunzhou Road, Kunming, Yunnan 650106, P.R. China

E-mail: youngjean1982@aliyun.com

Key words: peptide, MDA-MB-231 cell line, vector, transmembrane transduction, fusion protein delivery, tumor targeting therapy, breast cancer
PI-GST into MDA-MB-231 cells. Thus, PI could be modified to be a potential vector, and may contribute to the development of targeted therapeutic strategies for breast cancer.

\section{Introduction}

Human breast cancer is a malignant breast tumor that primarily affects females. Effective control of breast cancer has been hampered by a lack of specific tumor targets. One potential way to improve the specificity of targeting is to develop novel vectors that specifically bind to and are internalized by tumor cells.

In recent years, receptor-mediated endocytosis proteins, including vascularendothelial growth factor receptor(VEGFR), epidermal growth factor receptor (EGFR), human epidermal growth factor receptor 2 (HER2) and the Arg-Gly-Asp (RGD) motif (1-4), and protein transduction domains (including human immunodeficiency virus trans-activator of transcription protein and VP22) (5-7), have attracted considerable interest in the drug delivery field due to their ability to translocate across biological membranes. Evidence suggests that certain short peptides have promising intracellular delivery activity, and a number of these proteins have been modified and used as drug carriers in preclinical antitumor trials $(8,9)$. However, the low cell specificity of this approach limits its application in tumor-targeted therapy $(10,11)$. Dysregulation of oncogenes and tumor suppressor genes in tumor cells results in abnormal transcription processes, which in turn result in the expression of novel or uncovered ligands on the tumor cell surface.

Recently, Ivanenkov et al (12) used phage display to identify a novel peptide that showed a high affinity to HEp-2 human epithelial cells, but no affinity to other types of cells. Subsequently, peptide sequences with unique cell-type specificities have been reported. However, little attention has been focused on the potential uses of these peptides (13-15).

We hypothesized that tumor-targeting efficiency may be greatly improved with the availability of a cell membrane transduction peptides that are able to bind to tumor-specific receptors and provide a higher tumor cell internalization rate. This strategy may provide a novel addition to current 
antitumor approaches. In the present study, using phage display technology $(16,17)$, we attempted to select a tumor-targeting peptide with high cell specificity and delivery capacity. In order to study the transmembrane transduction mechanism of the peptide, the peptide was synthesized and labeled with fluorescein isothiocyanate (FITC) green fluorescence at its $\mathrm{N}$-terminus. The association of the specific internalization of the peptide into MDA-MB-231 cells with macropinocytosis and caveolin- and clathrin-mediated endocytosis was investigated. Our previous study found that a lung cancer cell line, Calu-1, also demonstrated an affinity for the peptide, similarly to the human breast cancer cell line MDA-MB-231 (18); thus, the present study investigated the hypothesis that major histocompatibility complex class I (MHC-I) antigen molecules and the cytomembrane proteins of these two cell lines may also be candidate proteins that are involved in the process of transmembrane transduction of PI. MHC-I antigen-mediated transmembrane transduction was investigated, and the membrane proteins of MDA-MB-231 and Calu-1 were extracted and compared by two-dimensional (2-D) electrophoresis (19) to identify those that were common to both cell lines and may be involved in the process of transmembrane transduction of PI. Further, to investigate the delivery efficiency of PI to specific cancer cells, PI-glutathione-S-transferase (GST) was constructed and the internalization of the fusion protein was visualized by immunofluorescence microscopy.

\section{Materials and methods}

Chemicals and reagents. The pC89 phage display library of random peptides was provided by Dr Alessandra Luzzago (Integrated Research Biotech Model, Rome, Italy). The RGD-integrin was supplied by Dr Peter J. Stambrook (Department of Cell Biology, Neurobiology, and Anatomy, Vontz Center for Molecular Studies, College of Medicine, University of Cincinnati, Cincinnati, OH, USA). Escherichia coli BL21 (DE3) were provided by Invitrogen (Thermo Fisher Scientific, Waltham, MA, USA). The plasmid pGEX-2T was maintained in the Key Laboratory of Translational Medicine of Cell Therapy Technology of Yunnan Province (Department of Internal Medicine Oncology, First Affiliated Hospital of Kunming Medical University, Kunming, Yunnan, China). The GST agarose affinity chromatography column was purchased from GE Healthcare Life Sciences (Tokyo, Japan). The mouse anti-Schistosoma japonicum GST monoclonal antibody was provided by Thermo Fisher Scientific (catalog no., MA4-004-1MG; dilution, 1:500). Horseradish peroxidase (HRP)-labeled rabbit anti-mouse IgG polyclonal antibody was also supplied by Thermo Fisher Scientific (catalog no., 61-6520; dilution, 1:1,000). ProteoPrep ${ }^{\circledR}$ Membrane Extraction Kit and sodium dodecyl sulfate (SDS) were products of Sigma-Aldrich (St. Louis, MO, USA). Polyclonal mouse anti-human MHC-I antibody (catalog no., ab76795; dilution, 1:500) was purchased from Abcam (Cambridge, MA, USA). RPMI-1640 medium, Dulbecco's modified Eagle's medium and fetal bovine serum were purchased from Gibco (Thermo Fisher Scientific). The human breast cancer cell lines MDA-MB-231, MDA-MB-435 and MCF-7, and all other tumor cell lines (HeLa, A431, SCC-29, Calu-1, Calu-3, GLC and U251) were purchased from the American Type Culture Collection.
Peptide synthesis. Using the manual solid-phase Fmoc method, PI (sequence, CASPSGALRSC) (18) was synthesized to determine whether the phage-coating protein was required for internalization into target cells. For cellular localization, the synthesized peptide was labeled with FITC at the N-terminus of PI (designated PI-FITC). Purification of the crude product was applied by reverse phase high-performance liquid chromatography (Agilent Technologies, Santa Clara, CA, USA), and identification of chemical structures was conducted by matrix-assisted laser desorption/ ionization time-of-flight mass spectrometry (JMS-S3000; Bruker Daltonic, Inc., Billerica, MA, USA).

Cell culture. MDA-MB-231 cells were cultured in Leibovitz's L-15 Medium (Gibco; Thermo Fisher Scientific) containing $100 \mathrm{ml} / 1$ fetal bovine serum and $50 \mathrm{ml} / 1 \mathrm{CO}_{2}$ at $37^{\circ} \mathrm{C}$. Calu-3 cells were cultured in Eagle's Minimum Essential Medium (American Type Culture Collection, Manassas, VA, USA) containing $150 \mathrm{ml} / 1$ fetal bovine serum at $37^{\circ} \mathrm{C}$ in $50 \mathrm{ml} / 1$ $\mathrm{CO}_{2}$. MDA-MB-435, HeLa, U251, MCF-7, SCC-29 and GLC cells were cultured and maintained in RPMI-1640 (Gibco; Thermo Fisher Scientific) supplemented with $100 \mathrm{ml} / \mathrm{l}$ fetal bovine serum at $37^{\circ} \mathrm{C}$ in $50 \mathrm{ml} / 1 \mathrm{CO}_{2}$. A431 cells were cultured in DMEM-H (Gibco; Thermo Fisher Scientific) containing $150 \mathrm{ml} / 1$ fetal bovine serum and $50 \mathrm{ml} / \mathrm{l} \mathrm{CO}_{2}$ at $37^{\circ} \mathrm{C}$. Calu-1 cells were cultured in McCoy's 5A (modified) medium (Gibco; Thermo Fisher Scientific) containing $100 \mathrm{ml} / 1$ fetal bovine serum and $50 \mathrm{ml} / 1 \mathrm{CO}_{2}$ at $37^{\circ} \mathrm{C}$.

Cell-type affinity assay of PI-FITC. MDA-MB-231 cells in the logarithmic phase were grown in a 96-well tissue culture plate for $12 \mathrm{~h}$, and cells were treated with PI-FITC at concentrations of 200, 500 and 1,000 ng/ml. RGD-integrin labeled with FITC was used as a control. The ability of PI-FITC to internalize into other breast cancer cells (MDA-MB-435 and MCF-7) and other solid tumor cells (HeLa, A431, SCC-29, Calu-3, GLC and U251) in the logarithmic phase was also tested by laser scanning confocal microscopy. For microscopy, the MDA-MB-231, MCF-7, HeLa, A431, SCC-29, Calu-3, GLC and U251 cells were treated with PI-FITC and RGD-integrin separately at concentrations of 200, 500 or $1,000 \mathrm{ng} / \mathrm{ml}$. The duration of incubation with PI-FITC and RGD-integrin was $12 \mathrm{~h}$. In this experiment, RGD-integrin was also labeled with FITC. Green fluorescence signals of FITC were observed by scanning confocal microscopy (Zeiss LSM 800; Carl Zeiss AG, Oberkochen, Germany).

Concentration-, temperature- and time-dependence of PI internalization to $M D A-M B-231$ cells. As it is possible that the affinity of PI-FITC for MDA-MB-231 may be influenced by varying PI-FITC concentrations, incubation times and temperatures, experiments were conducted to investigate the effects of these parameters. For the concentration-dependence experiment, $5 \times 10^{4}$ MDA-MB-231 cells in the logarithmic phase were grown in 24-well tissue culture plates and divided into four subgroups; $0,2,5$ or $10 \mu \mathrm{mol} / 1$ of PI-FITC was added to each subgroup, respectively. Following $1 \mathrm{~h}$ of incubation, cells were harvested, washed in phosphate-buffered saline (PBS; Beijing Jimei Biotechnology Co., Ltd., Beijing, China), fixed in formaldehyde (Beijing Jimei Biotechnology Co., Ltd.) 
and permeabilized with Triton X-100 (Sigma-Aldrich). The PI-FITC distribution of each subgroup was observed by flow cytometry (FACSCanto II; catalog no., 338960; BD Biosciences, Franklin Lakes, NJ, USA).

For the temperature-dependence experiment, $5 \times 10^{4}$ MDA-MB-231 cells in the logarithmic phase were grown in three 24 -well tissue culture plates, $2 \mu \mathrm{mol} / 1$ of PI-FITC was added to each plate, and the plates were incubated for $1 \mathrm{~h}$ at temperatures of 4,25 or $37^{\circ} \mathrm{C}$, respectively. Following incubation, flow cytometry was used to observe the results. MDA-MB-231 cells without PI-FITC were used as a control.

In the time-dependence experiment, 5x10 4 MDA-MB-231 cells in the logarithmic phase were grown in three 24-well tissue culture plates, $2 \mu \mathrm{mol} / 1$ of PI-FITC was added to each plate, and the plates were incubated for 1,6 or $12 \mathrm{~h}$. A plate containing MDA-MB-231 cells without PI-FITC was used as a control, and flow cytometry was applied to analyze the results.

Transmembrane transduction inhibition analysis. To investigate whether the transmembrane transduction mechanism of PI was associated with macropinocytosis or caveolin- or clathrin-mediated endocytosis, three inhibitors of cytomembrane transport, consisting of amiloride (Sanofi China, Hangzhou, China) (19), methyl- $\beta$-cyclodextrin (Santa Cruz Biotechnology, Inc., Dallas, TX, USA) (20) and chlorpromazine (Amresco, Cleveland, $\mathrm{OH}$, USA) (21) were incubated with MDA-MB-231 cells. An MTT assay (Sigma-Aldrich) was used to determine the toxicity of these membrane channel inhibitors to MDA-MB-231 cells.

For each experiment, $5 \times 10^{4}$ MDA-MB-231 cells in the logarithmic growth phase were grown in 24-well tissue culture plates and equally divided into three subgroups, designated A, $\mathrm{B}$ and $\mathrm{C}$; cells in each subgroup were treated with PI-FITC, PI-FITC plus an inhibitor (amiloride, methyl- $\beta$-cyclodextrin or chlorpromazine), or normal saline, respectively. The distribution of PI-FITC in MDA-MB-231 cells in each experimental group was subsequently investigated by flow cytometry.

MHC-I antigen analysis. There are 250,000 molecules of each type of human leukocyte antigen (HLA) on the surface of each human cell (22). Our preliminary studies indicated that the human lung cancer cell line Calu-1 had high affinity to PI, similarly to MDA-MB-231 cells. Therefore, the present study compared the MHC-I antigen molecules of these two cell lines and further analyzed whether the MHC-I antigen molecules may be involved in transmembrane transduction. PI-FITC was added to the experimental cultural system of MDA-MB-231 cells and positive cultural system of Calu-1 cells, and both were incubated with an anti-MHC-I antibody. After the MHC-I molecules on the cell surfaces were blocked by the anti-MHC-I antibody, the distributions of PI-FITC in these two cell types were detected under fluorescence microscopy (DM-IL; Leica, Wetzlar, Germany). DNA from MDA-MB-231 and Calu-1 cells were extracted using the QIAamp DNA Mini Kit (Qiagen, Hilden, Germany), according to the manufacturer's protocol. HLA-A and -B DNA fragments were amplified and sequence-specific primer (SSP)-polymerase chain reaction (PCR) was applied to analyze the MHC-I antigen molecules. The MHC-I antigen molecules were analyzed using the AB/DR/DQ SSP UniTray-96 kit from Texas BioGene, Inc. (Richardson, TX, USA). PCR was performed for 21 cycles as follows: Denaturation at $96^{\circ} \mathrm{C}$ for $25 \mathrm{sec}$; annealing at $65^{\circ} \mathrm{C}$ for $50 \mathrm{sec}$; and extension at $72^{\circ} \mathrm{C}$ for $45 \mathrm{sec}$. Extension was then performed at $72^{\circ} \mathrm{C}$ for another $10 \mathrm{~min}$. Separation and determination of PCR products were conducted by electrophoresis. The ProFlex PCR machine was provided by Applied Biosystems (Thermo Fisher Scientific).

Membrane protein analysis. To investigate whether the transmembrane transduction mechanism of PI is associated with the same membrane proteins in the MDA-MB-231 and Calu-1 cell lines, the membrane proteins were extracted from the two types of cell using the ProteoPrep Membrane Extraction Kit, according to the instructions, and were compared by 2-D electrophoresis (23). The protein profiles of the two cell lines were analyzed by PDQuest Software for 2-D Gel Analysis, version 7.0 (Bio-Rad Laboratories, Inc., Hercules, CA, USA), with the help of the Institute of Medical Biology, Chinese Academy of Medical Sciences (Beijing, China).

Construction and expression of the recombinant GST fusion protein PI-GST. The encoding region (5'-TGCGCATCCCCA TCTGGCGCCCTTCGTTGTTGC-3') of PI was synthesized with the addition of a BamHI site upstream and an EcoRI site downstream by Takara Bio Inc. (Otsu, Japan). The synthesized product was ligated into the pGEX-2T plasmid at the BamHI and EcoRI sites. The insert, cut with the restriction enzymes (Thermo Fisher Scientific), was subject to SDS-polyacrylamide gel electrophoresis (PAGE) and was identified by DNA sequence analysis. The Escherichia coli strain BL21 (DE3) was transformed with the recombinant plasmid pGEX-2T-PI or vector alone using the $\mathrm{CaCl}_{2}$ transformation method (24) and then grown in a lysogeny broth solution (Sigma-Aldrich) containing $100 \mathrm{mg} / \mathrm{ml}$ of ampicillin (Amresco) at $37^{\circ} \mathrm{C}$. Isopropyl- $\beta$-D-thiogalactopyranoside (IPTG; Amresco), an inducer of $\beta$-galactosidase activity in bacteria, was added to a final concentration of $1 \mathrm{mM}$, and the solution was incubated at $37^{\circ} \mathrm{C}$ for 1-6 h. Following IPTG induction, bacterial pellets were obtained by centrifugation at $2,000 \mathrm{x}$ g at $4^{\circ} \mathrm{C}$.

Western blot analysis of PI-GST. Fusion protein products expressed in bacterial pellets were released by ultrasonic membrane rupture and centrifuged at $5,000 \mathrm{x} \mathrm{g}$ at $4^{\circ} \mathrm{C}$. The supernatant was collected and purified by GST affinity column. One-dimensional SDS-PAGE was performed using $12.5 \%$ (wt/vol) polyacrylamide gels. For immunoblotting, proteins were transferred from polyacrylamide gels to Immobilon ${ }^{\mathrm{TM}}$ polyvinylidene difluoride membranes (Sigma-Aldrich) using Tris-glycine electroblotting buffer (Sigma-Aldrich) at 15-20 mA overnight. The membranes were blocked with $5 \%$ evaporated skimmed milk for $30 \mathrm{~min}$ at $37^{\circ} \mathrm{C}$ to prevent non-specific binding. The membranes were then incubated with the primary mouse anti-GST monoclonal antibody (dilution, 1:500) overnight at $4^{\circ} \mathrm{C}$ and with the secondary HRP-conjugated goat anti-mouse $\operatorname{IgG}$ (dilution, 1:1,000) for $30 \mathrm{~min}$ at $37^{\circ} \mathrm{C}$. The expressed product was identified by treating with dextran sulfate and 4-methylbenzamide for $\sim 5 \mathrm{~min}$.

Purification of PI-GST. The fused protein products in the supernatant fraction were purified on GST-sepharose columns. Purification was conducted as described by the manufacturer. Briefly, $100 \mathrm{ml}$ of sample was centrifuged to remove any 
undissolved membranes and cellular debris before being added to the column. Triton X-100 was then added to the collected supernatants. The column was washed with 5-10 bed volumes of PBS to remove azide. The gel bed was equilibrated with 3-5 bed volumes of PBS containing 1\% Triton X-100. Subsequently, the sample was added to the prepared column. The flow-through was collected as a control. The column was washed with 10 bed volumes of PBS until no protein could be detected in the elution. The setup included sufficient Eppendorf tubes for a standard curve with $0,5,10,15,20$ and $25 \mathrm{~g}$ of bovine serum albumin (BSA) and the samples to be tested. The harvest was subject to western blotting for identification, as described.

Transduction activity of fusion protein. MDA-MB-231 cells were grown in 24-well tissue culture plates at $37^{\circ} \mathrm{C}$ for $12 \mathrm{~h}$. Cells were incubated with PI-GST at 20, 50, 100, 200 or $500 \mathrm{ng} / \mathrm{ml}$ for $8-12 \mathrm{~h}$ at $37^{\circ} \mathrm{C}$. The cultured cells were fixed in $10 \%$ Triton X-100 for $10 \mathrm{~min}$ and treated with buffer $\left(1 \% \mathrm{BSA}, 0.025 \% \mathrm{NaN}_{3}, 0.1 \%\right.$ saponin) for $15 \mathrm{~min}$. The GST monoclonal antibody (dilution, 1:800) was added to the cells for $30 \mathrm{~min}$ at $4^{\circ} \mathrm{C}$. The cells were then incubated with HRP-labeled rabbit anti-mouse IgG antibody (dilution, 1:1,000) for $30 \mathrm{~min}$ at $37^{\circ} \mathrm{C}$, subsequent to washing the cells 3 times. Internalization was visualized by immunofluorescence microscopy (DM-IL; Leica). MDA-MB-231 cells incubated with GST were used as a blank control.

\section{Results}

Internalization assay of synthesized peptides in MDA-MB-231 and other cell lines. The green fluorescence signal of PI-FITC was easily detectable in each MDA-MB-231 cell after 12-48 h of incubation, indicating that PI-FITC was efficiently taken up by MDA-MB-231 cells; the internalized PI was predominantly located in the cytoplasm or around the nuclear membrane. No green fluorescence signal could be observed in the MDA-MB-231 cells without PI-FITC (Fig. 1). Compared with RGD-integrin labeled with FITC, the internalization activity of PI in MDA-MB-231 cells was similar (Fig. 2). In contrast to MDA-MB-231 cells incubated with PI-FITC, no fluorescence signal was observed in the other breast cancer cell lines, MDA-MB-435 and MCF-7 (Fig. 3), and the synthesized peptides exhibited no affinity to the other types of tumor cell, HeLa, A431, SCC-29, Calu-3, GLC and U251 (Table I).

Concentration-, temperature- and time-dependence of PI internalization to $M D A-M B-231$. Flow cytometry results revealed an increase in cell-associated fluorescence with increasing concentration of PI, indicating that the concentration of PI is associated with its internalization (Fig. 4A); both the number of MDA-MB-231 cells and the concentration of PI-FITC were influencing factors on the internalization of PI-FITC. However, the incubation temperature and time had little influence on the internalization of PI (Fig. 4B and C)

Membrane channel inhibition experiments. Following the addition of amiloride, methyl- $\beta$-cyclodextrin and chlorpromazine to the MDA-MB-231 cells, MTT results indicated that these three channel inhibitors had no significant toxicity to the growth of MDA-MB-231 cells (Fig. 5). In the amiloride and methyl- $\beta$-cyclodextrin experimental groups,
Table I. Results of an internalization assay of the synthesized peptide, PI, into various cell lines.

Cell line Fluorescence signal of PI-FITC

MDA-MB-231
MDA-MB-435
MCF-7
Hela
A431
SCC-29
Calu3
GLC
U251

PI, peptide (CASPSGALRSC); FITC, fluorescein isothiocyanate.

Table II. Analysis of the alleles of HLA-I in the MDA-MB-231 and Calu-1 cell lines.

\begin{tabular}{lllll}
\hline \multicolumn{2}{c}{ MDA-MB-231 } & & \multicolumn{2}{c}{ Calu-1 } \\
\cline { 5 - 6 } HLA-A & HLA-B & & HLA-A & HLA-B \\
\hline A*02:01 & B*41:01 & & A*26:01 & B*44:03 \\
A*02:02 & B*41:03 & & A*26:02 & B*44:04 \\
A*02:03 & B*41:05 & & A*26:08 & B*44:05 \\
A*24:02 & B*40:02 & & A*29:01 & B*15:01 \\
A*24:03 & B*40:03 & & A*29:02 & B*15:04 \\
A*24:04 & B*40:04 & & A*29:03 & B*15:27
\end{tabular}

Six alleles of HLA-A site were found in MDA-MB-231 and Calu-1 cells, and six alleles of the HLA-B site were also found in MDA-MB-231 and Calu-1 cells. Among all these alleles, no similar allele was found. HLA, human leukocyte antigen.

flow cytometry results suggested that the distribution of PI-FITC in MDA-MB-231 cells of each group had decreased (Fig. 6A and B). However, in the chlorpromazine group, no significant change in intracellular distribution was observed (Fig. 6C).

MHC-I antigen analysis. After the MHC-I antigen had been blocked in the MDA-MB-231+PI-FITC and Calu-1+PI-FITC culture systems, the distributions of PI-FITC in the cells of the two culture systems were consistent with the control groups without MHC-I antibody. DNA samples of MDA-MB-231 cells and Calu- 1 cells were detected by PCR-SSP. The results revealed that none of the same MHC-I antigen molecules could be detected. This indicated that the MHC-I antigen molecules of the two cell lines may not be involved in the process of transmembrane transduction of PI (Table II).

Membrane protein comparison by 2-D electrophoresis map. Following the extraction of membrane proteins from the two cell lines, 2-D electrophoresis of each sample was repeated four times, and the protein profiles of the two cell types were 


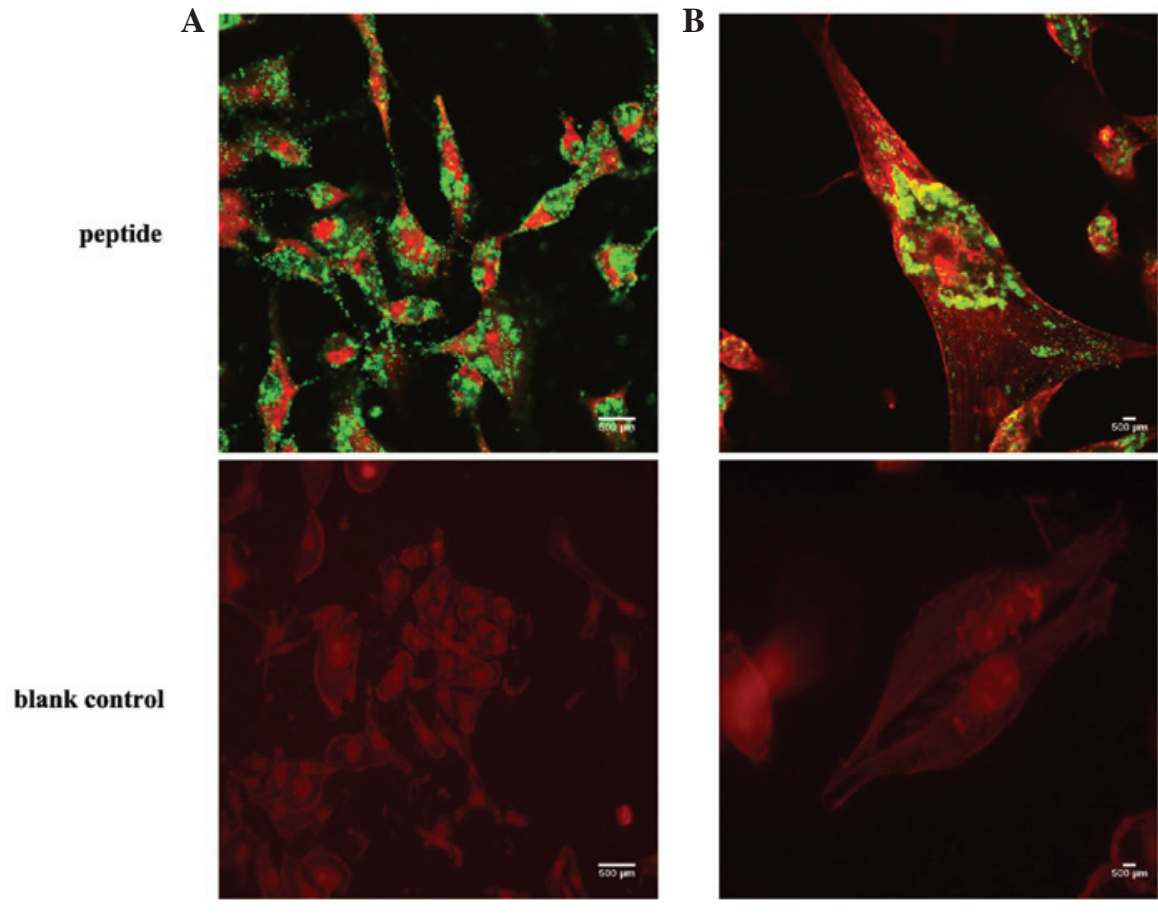

Figure 1. Internalization of PI-FITC in MDA-MB-231 cells observed by laser scanning confocal microscopy. The upper panels show MDA-MB-231 cells treated with PI-FITC. The lower panels show MDA-MB-231 cells without PI-FITC. MDA-MB-231 cells were observed as red fluorescence, and the green fluorescence represents the signal from PI-FITC. (A) x200 and (B) x600 magnification. PI, synthesized peptide (CASPSGALRSC); FITC, fluorescein isothiocyanate.

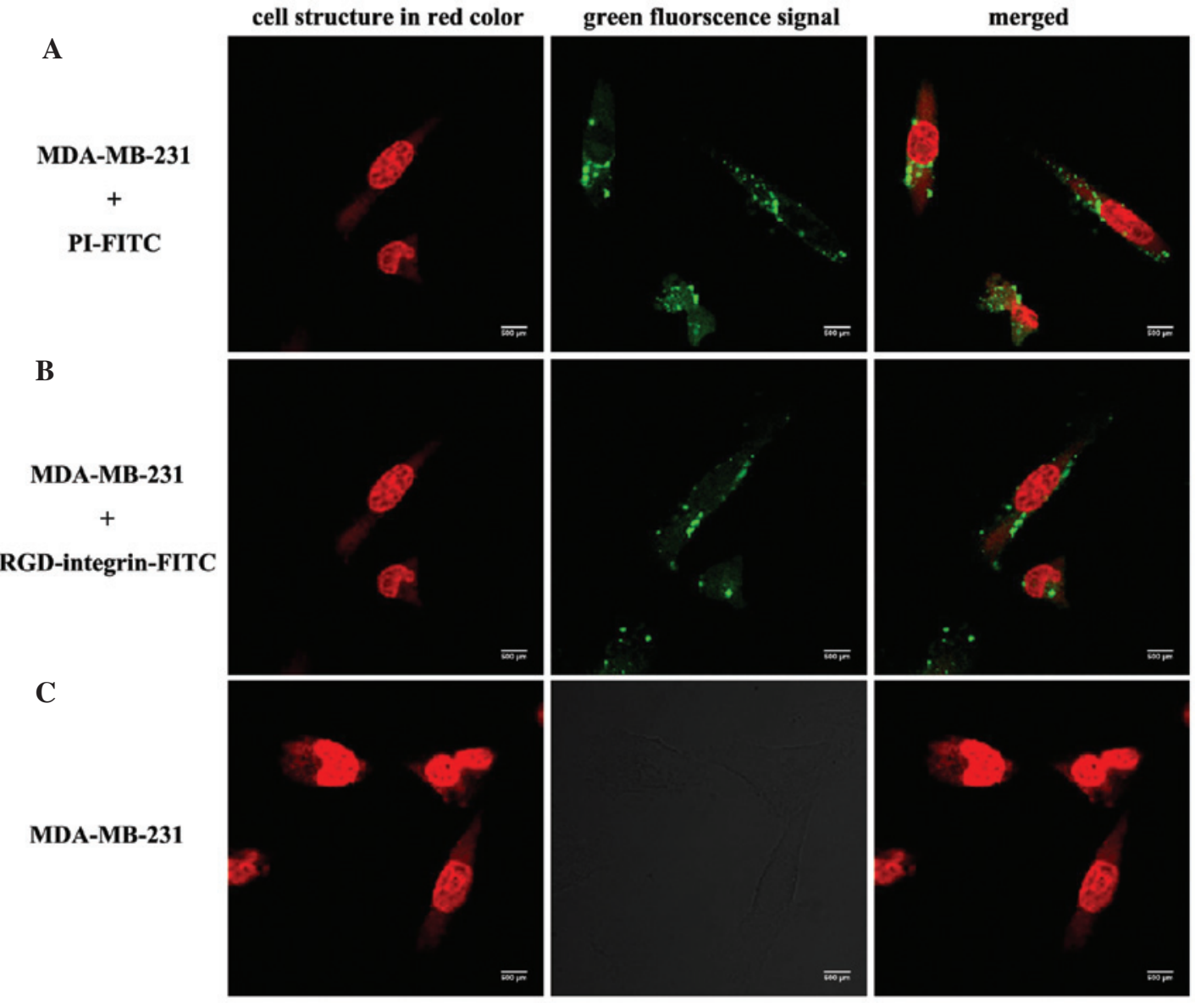

Figure 2. Cell-type affinity assay of PI-FITC observed by laser scanning confocal microscopy (magnification, x200). Left images show representative MDA-MB-231 cells in red. Middle images show green fluorescence signal of FITC. Right images show the merged picture of the cells and the green fluorescence. (A) MDA-MB-231 cells incubated with PI-FITC; (B) MDA-MB-231 cells incubated with RGD-integrin-FITC; (C) MDA-MB-231 cells only (no green fluorescence signal was detected). PI, synthesized peptide (CASPSGALRSC); FITC, fluorescein isothiocyanate; RGD, Arg-Gly-Asp motif. 


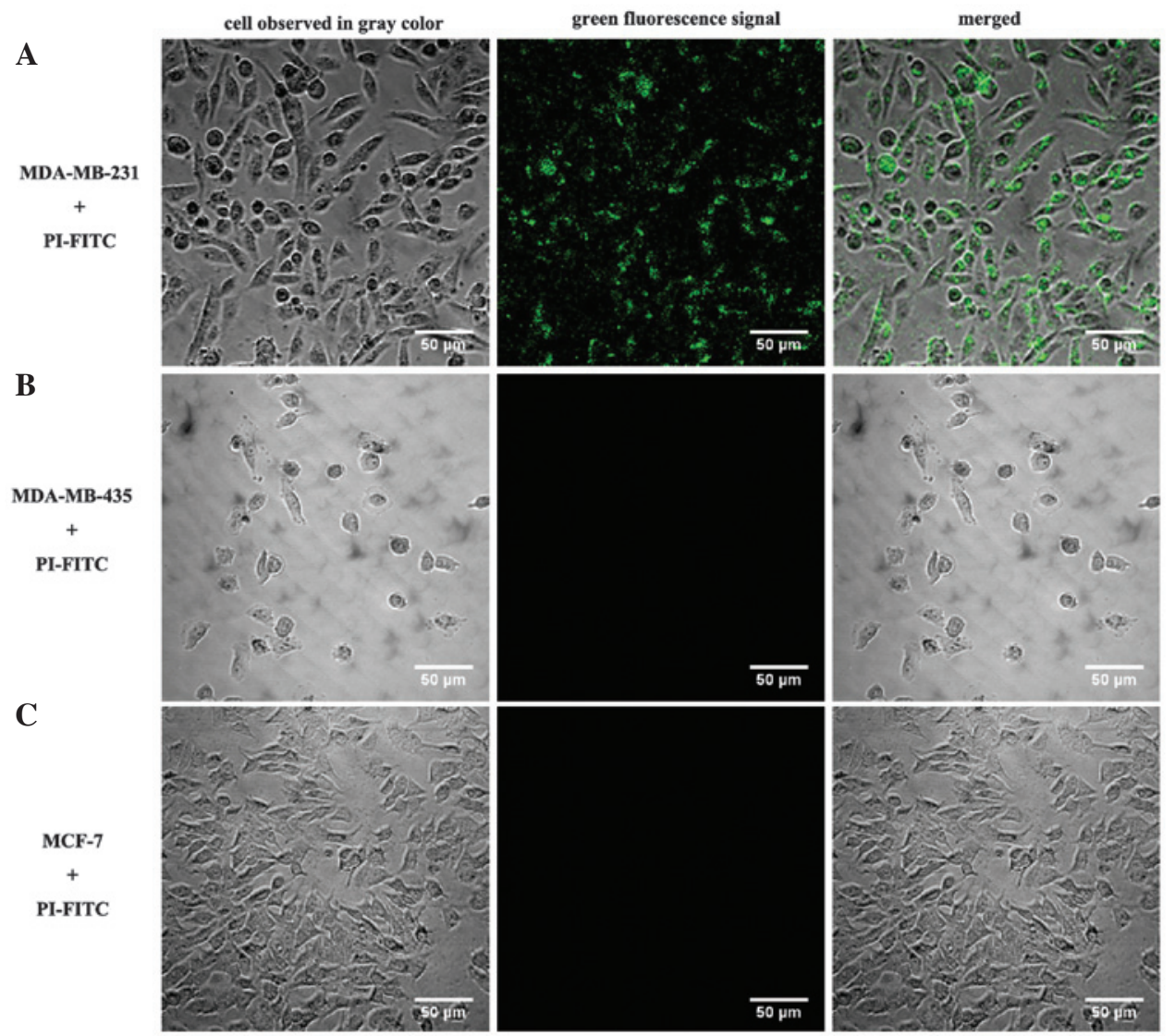

Figure 3. Cell-specific affinity analysis of PI-FITC with various human breast cancer lines under laser scanning confocal microscopy (magnification, $\mathrm{x} 40$ ). Left images show representative areas of MDA-MB-231 cells in gray; middle images show representative areas of FITC green fluorescence; right panels show the merged images of the cells and the green fluorescence. (A) MDA-MB-231 cells incubated with PI-FITC; (B) MDA-MB-435 and (C) MCF-7 cells incubated with PI-FITC (no green fluorescence was observed). PI, synthesized peptide (CASPSGALRSC); FITC, fluorescein isothiocyanate.
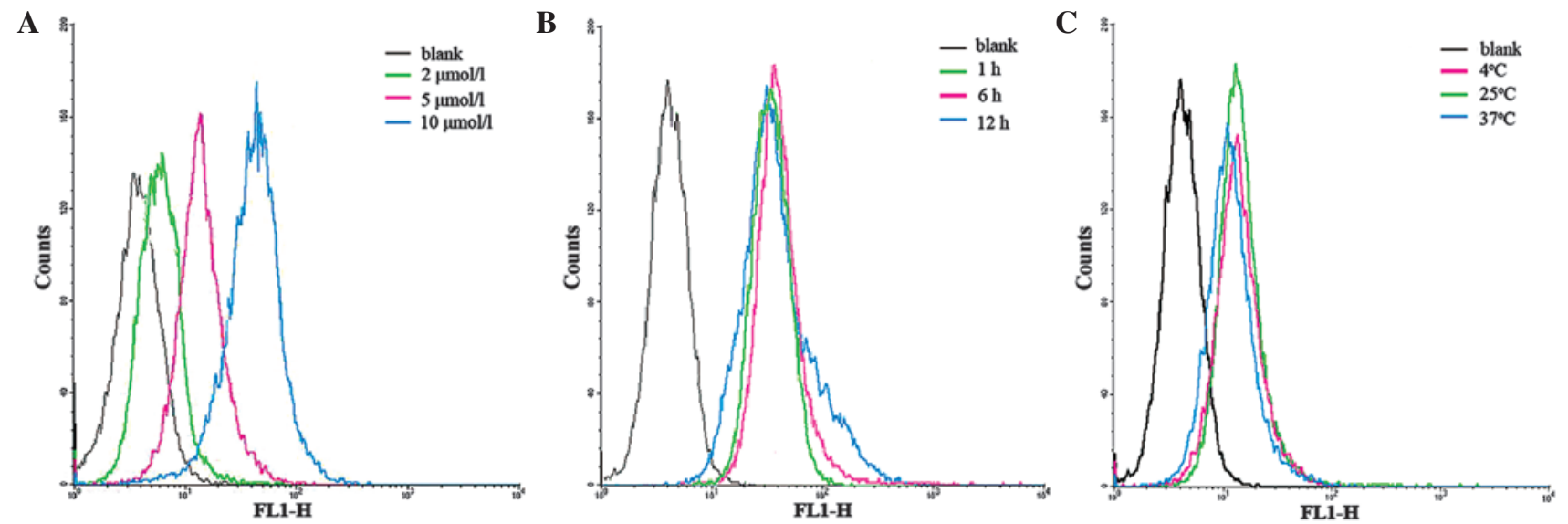

Figure 4. Concentration-, temperature- and time-dependence of PI internalization to MDA-MB-231 cells. (A) Distribution of PI-FITC in MDA-MB-231 cells following incubation with various concentrations of PI-FITC; results indicated an increase in cell-associated fluorescence suggesting that the concentration of PI is associated with its internalization. (B) Distribution of PI-FITC in MDA-MB-231 cells following incubation with PI-FITC for various durations; the internalization of PI-FITC in MDA-MB-231 cells was similar following 1, 6 and $12 \mathrm{~h}$ of incubation. (C) Distribution of PI-FITC in MDA-MB-231 cells after different incubation temperatures; the internalization of PI-FITC in MDA-MB-231 cells was similar after incubating at 4,25 and $37^{\circ} \mathrm{C}$. PI, synthesized peptide (CASPSGALRSC); FITC, fluorescein isothiocyanate.

analyzed by PDQuest Software for 2-D Gel Analysis. Around 260 protein spots of each sample were detected, and a total of 11 common protein spots were identified (Fig. 7).

Expression of PI-GST. Expression of PI-GST and GST was induced by IPTG, and crude bacterial extracts were analyzed by SDS-PAGE. Two constructs produced protein of the expected molecular mass, 26-27 kDa (Fig. 8). Western blot analysis of the crude extract indicated that the fusion protein reacted with an antibody against GST. All data demonstrated that the GST-fused protein PI-GST was present. 


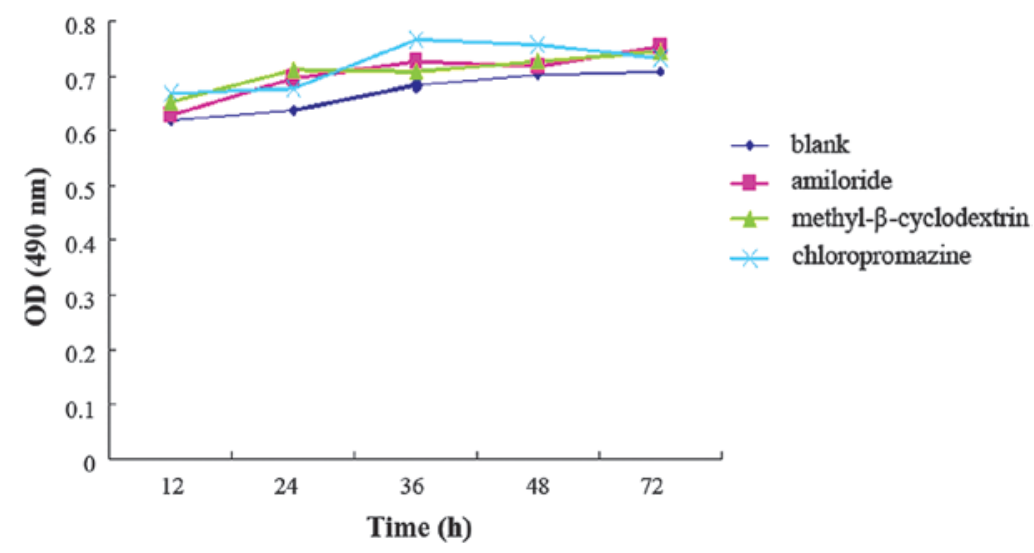

Figure 5. Growth curve of MDA-MB-231 cells treated with amiloride, methyl- $\beta$-cyclodextrin and chlorpromazine. The growth states of all the cells of the four groups were consistent, indicating that they were largely unaffected by the three substances. OD, optical density.

A

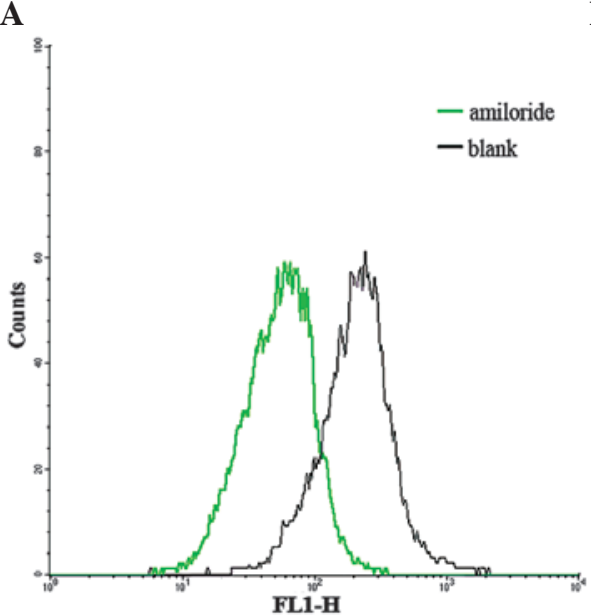

B

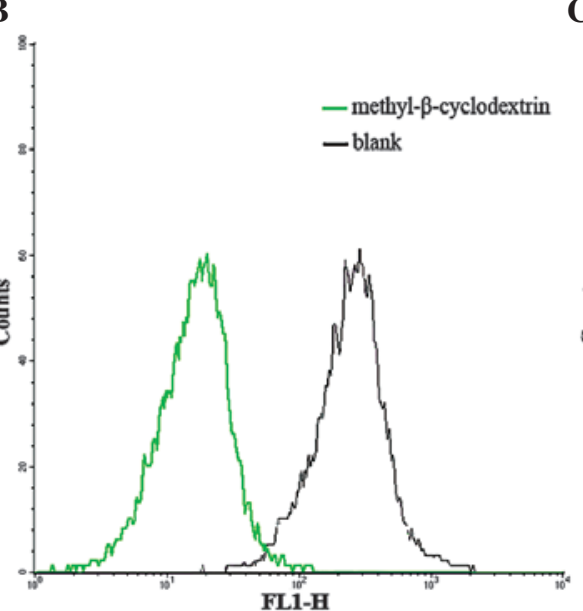

C

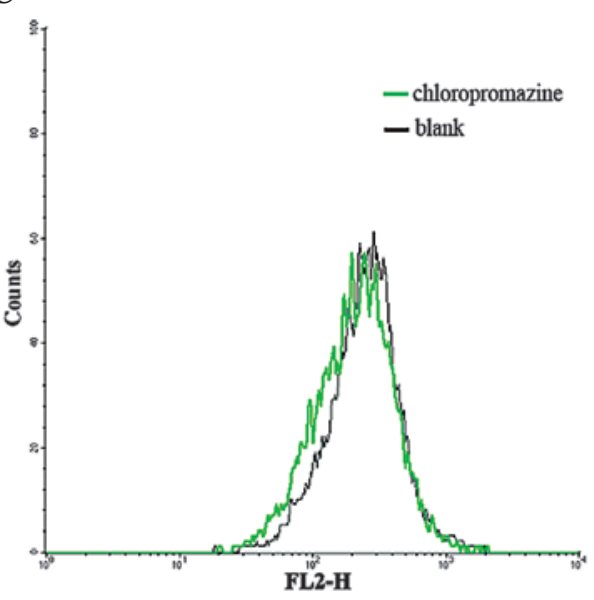

Figure 6. Membrane channel inhibition experiments. (A) Distribution of PI-FITC in MDA-MB-231 cells following addition of amiloride; compared with the MDA-MB-231+PI-FITC culture system (blank), amiloride led to a decrease in the distribution of PI-FITC in MDA-MB-231 cells. (B) Distribution of PI-FITC in MDA-MB-231 cells following addition of methyl- $\beta$-cyclodextrin; compared with the blank culture system, methyl- $\beta$-cyclodextrin led to a decrease in the distribution of PI-FITC in MDA-MB-231 cells. (C) Distribution of PI-FITC in MDA-MB-231 cells following the addition of chlorpromazine; the distribution of PI-FITC in MDA-MB-231 cells was similar in the chlorpromazine and blank groups. PI, synthesized peptide (CASPSGALRSC); FITC, fluorescein isothiocyanate.

A

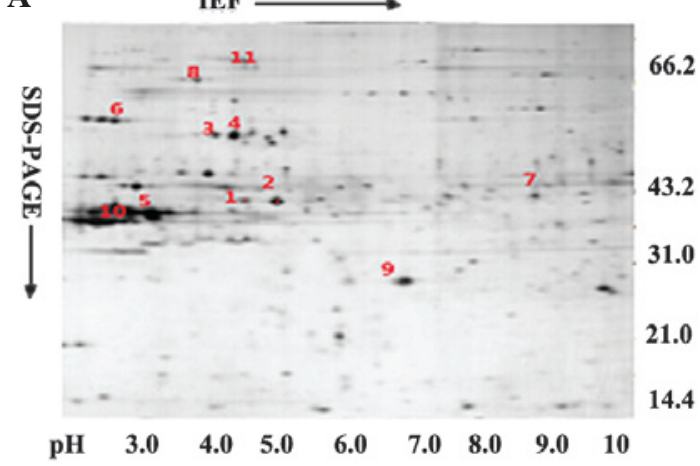

B

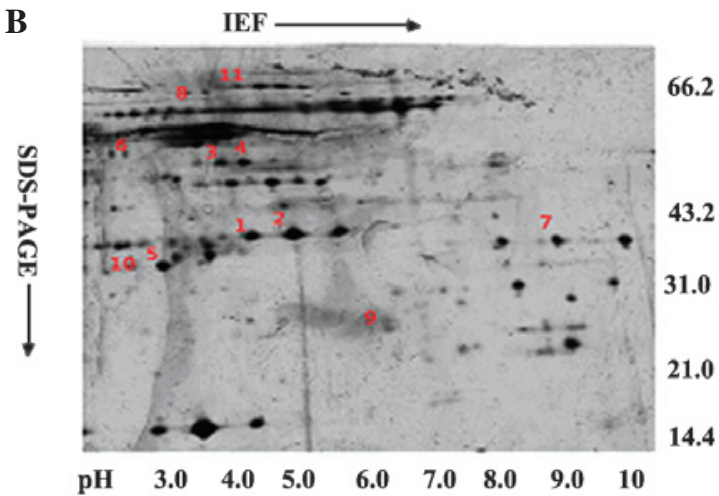

Figure 7. Membrane protein comparison by two-dimensional electrophoresis map: (A) MDA-MB-231 cells and (B) Calu-1 cells. A total of 11 common protein spots were observed. SDS-PAGE, sodium dodecyl sulfate polyacrylamide gel electrophoresis; IEF, isoelectric focusing.

Delivery efficiency of fusion protein into target cells. In the presence of increasing concentrations $(20-500 \mathrm{ng} / \mathrm{ml})$ of the PI-GST fusion protein for $72 \mathrm{~h}$ at $37^{\circ} \mathrm{C}$, cellular viability was $95 \%$. Cellular toxicity was not observed in the experimental cells. At $200 \mathrm{ng} / \mathrm{ml}$, the optimal concentration of the peptide PI-GST was taken up by MDA-MB-231 cells efficiently. In the initial 12-18 h culturing period, the fluorescence intensity of PI-GST in MDA-MB-231 cells was 


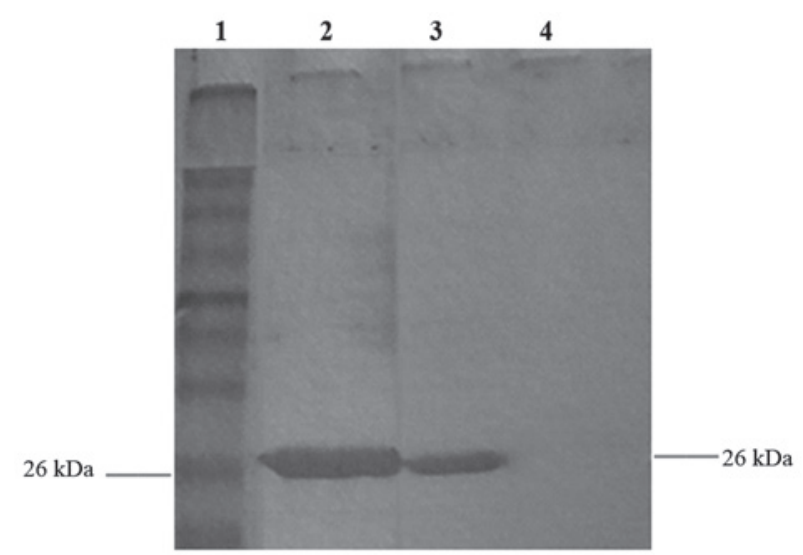

Figure 8. Western blotting of the fusion protein expressed in E. coli BL21 (DE3). Lane 1, marker; lane 2, GST; lane 3, PI-GST; lane 4, blank control. GST, glutathione-S-transferase; PI, synthesized peptide (CASPSGALRSC).

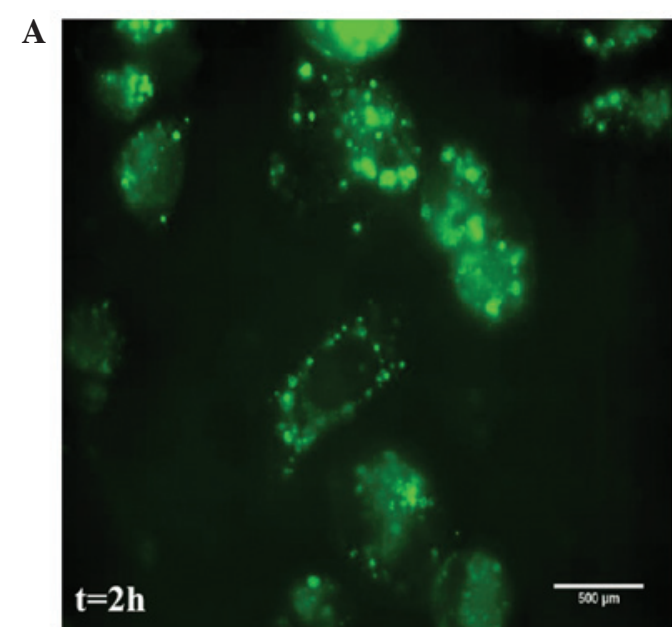

C

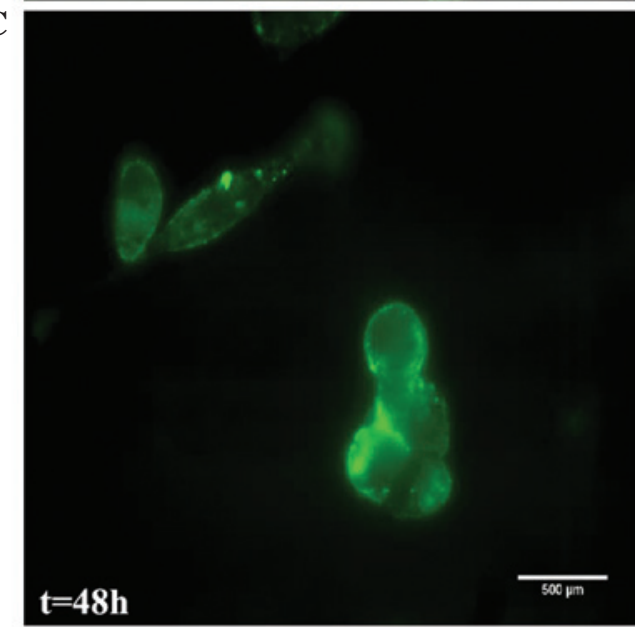

B
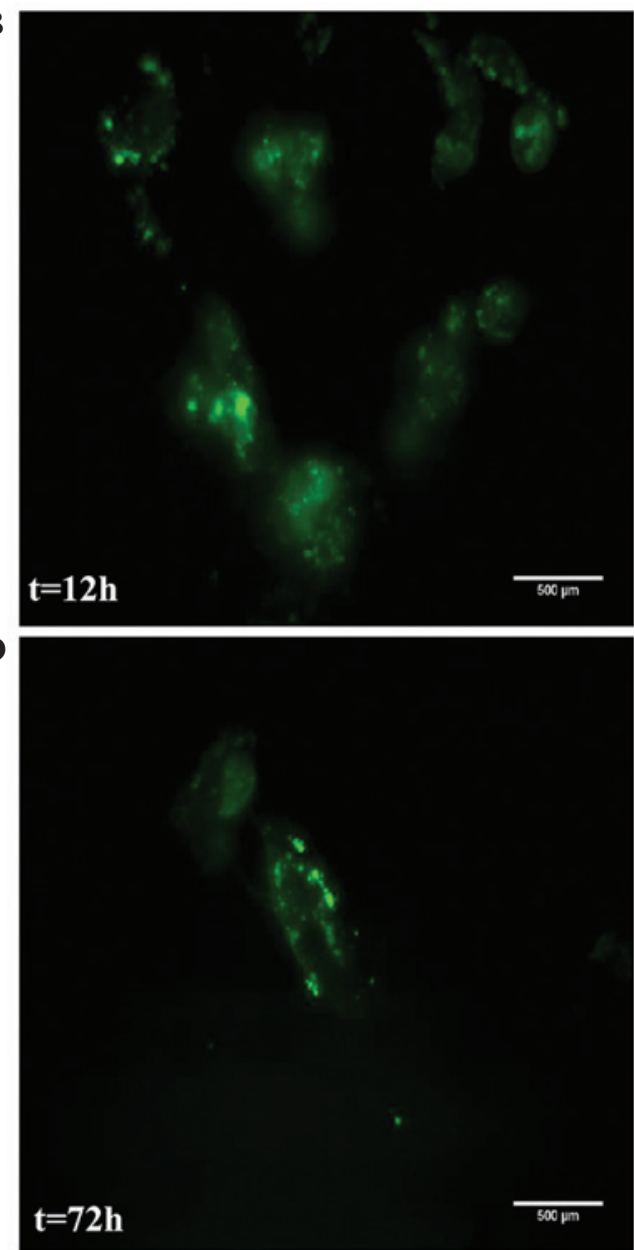

Figure 9. Dynamic imaging of the delivery efficiency of the peptide observed by fluorescence microscopy (magnification, x200). (A) MDA-MB-231 cells after $2 \mathrm{~h}$ of incubation with PI-GST; fluorescence microscopy indicated that PI is able to deliver GST protein into MDA-MB-231; (B) after 12 h, the fusion protein was detected in the cytoplasm; (C) $48 \mathrm{~h}$ and (D) $72 \mathrm{~h}$ later, the signal of the fusion protein in the majority of cells became weak and eventually disappeared. PI, synthesized peptide (CASPSGALRSC); GST, glutathione-S-transferase.

as strong as that observed for PI itself (Fig. 9A); a strong intracellular PI-GST signal was observed in the cytoplasm after $12 \mathrm{~h}$ (Fig. 9B). After 48-72 h, the signal of the fusion protein in the majority of the cells became weak and disappeared (Fig. 9C and D).

\section{Discussion}

Breast cancer is the most commonly diagnosed cancer in women and is the second leading cause of cancer-associated mortality (25). The risks of local recurrence, metastasis and 
drug resistance of tumors remain difficult to overcome and control (26).

Recent targeted and biological therapies reflect novel and promising improvements in the treatment of breast cancer; for example, drugs including trastuzumab, bevacizumab, gefitinib and celecoxib have been designed to focus on identified tumor-associated targets and have demonstrated promising results in clinical practice (27-32). However, these therapeutic targets are expressed in numerous tissues, suggesting that the cellular specificity is extremely low and may cause potential side effects $(33,34)$. Therefore, the development of agents with cancer cell selectivity is urgently required to improve targeted therapy.

It has been reported that use of the phage display system allows selection of peptide sequences with unique cell-type specificity $(35,36)$. We hypothesized that a breast cancer-specific peptide may also be screened through the phage display system. The strategy adopted for the current study was to screen a breast cancer-specific peptide, evaluate whether it has potential as a specific delivery system for tumor-targeting therapy, and investigate the delivery mechanism.

The synthesized PI peptide (CASPSGALRSC) was incubated with MDA-MB-231 cells in order to investigate it as a peptide with tumor cell specificity. To improve comparability, RGD-integrin, which is known to be recognized and internalized by various types of human cells $(37,38)$, was set as a control. The synthesized PI exhibited no affinity to other cancer cell lines MDA-MB-435, MCF-7, HeLa, A431, SCC-29, Calu-3, GLC and U251. These affinity experiments suggested that PI was a novel type of transmembrane peptide with MDA-MB-231 cell specificity. Moreover, PI has no sequence similarity to RGD-integrin or to any other protein sequence available in various protein databases.

To further analyze the transduction mechanism of PI, three inhibitors of cytomembrane transport (amiloride, methyl- $\beta$-cyclodextrin and chlorpromazine) were used to investigate whether the specific internalization of the peptide into MDA-MB-231 cells is associated with macropinocytosis, caveolin-mediated endocytosis or clathrin-mediated endocytosis, using flow cytometry. The results revealed that the transduction of the peptide into the cell is partially mediated by macropinocytosis and caveolin-mediated endocytosis. Our initial study established that the lung cancer cell line Calu-1 also demonstrated high affinity to the peptide, similarly to MDA-MB-231 (39). Thus, we hypothesized that MHC-I antigen molecules may mediate transmembrane transduction, and the cytomembrane proteins of the two cell lines may be involved in the process of transmembrane transduction of PI. In the present study, MHC-I antigen molecules and the cytomembrane proteins of the two cell lines were investigated. The results revealed that MHC-I antigen molecules of MDA-MB-231 and Calu-1 share no common alleles, which indicated that these MHC-I antigen molecules have no association with the transmembrane transduction of PI. The results of 2-D electrophoresis identified 11 proteins spots that were common to MDA-MB-231 and Calu-1 cells, which may also be candidates for the transmembrane transduction of PI.

Given the notable cellular specificity of PI, its use as a vector for a therapeutic protein delivery may be a practical way to improve targeting efficiency in tumor therapy. To investigate the ability of PI to deliver a potential therapeutic protein, GST was introduced to represent an exogenous protein. GST was fused with PI and was used as a marker to assess the protein transduction ability of PI in vitro. PI was observed to be capable of successfully delivering GST into the cytoplasm of MDA-MB-231 cells, and the exogenous protein did not degrade for $\geq 72 \mathrm{~h}$. Significant immunofluorescence signals for PI-GST were observed in the MDA-MB-231 cell cytoplasm, while no GST signal was observed in MDA-MB-231 cells. Thus, it may be concluded that PI is able to deliver an exogenous protein of at least $26 \mathrm{kDa}$ into MDA-MB-231 cells. This transduction procedure is also cell-specific, which was confirmed by treating different human breast cancer cell lines with PI-GST. Additionally, PI and PI-GST did not lead to any cytotoxic effects when the cells were maintained in culture medium for $72 \mathrm{~h}$. Thus, the current research shows that PI has the necessary features to be an efficient drug delivery vector for targeted cancer therapy.

Taken together, our studies may lead to the discovery of a novel tumor cell-specific receptor and provide a new therapeutic target for cancer treatment and diagnosis (40). Such a peptide may serve as a specific vehicle for tumor-targeting therapy and may complement other molecular therapeutic approaches for localized and systemic application. Further research is required in this area.

\section{Acknowledgements}

This work was supported by the National Natural Science Foundation of China (no. 30860330), Science and Technology Platform Construction Project of Yunnan Province (no. 2007DA006) and Applied Basic Research Project of Yunnan Province (no. 2009CC023).

\section{References}

1. Cooke SP, Boxer GM, Lawrence L, Pedley RB, Spencer DI, Begent RH and Chester KA: A strategy for antitumor vascular therapy by targeting the vascular endothelial growth factor: Receptor complex. Cancer Res 61: 3653-3659, 2001.

2. Miller CR, Buchsbaum DJ, Reynolds PN, Douglas JT, Gillespie GY, Mayo MS, Raben D and Curiel DT: Differential susceptibility of primary and established human glioma cells to adenovirus infection: Targeting via the epidermal growth factor receptor achieves fiber receptor-independent gene transfer. Cancer Res 58: 5738-5748, 1998

3. Baselga J, Norton L, Albanell J, Kim YM and Mendelsohn J: Recombinant humanized anti-HER 2 antibody (Herceptin) enhances the antitumor activity of paclitaxel and doxorubicin against HER2 overexpressing human breast cancer xenografts. Cancer Res 58: 2825-2831, 1998.

4. Ruoslahti E and Pierschbacher MD: New perspectives in cell adhesion: RGD and integrins. Science 238: 491-497, 1987.

5. Frankel AD and Pabo CO: Cellular uptake of the tat protein from human immunodeficiency virus. Cell 55: 1189-1193, 1988.

6. Green M and Loewenstein PM: Autonomous functional domains of chemically synthesized human immunodeficiency virus tat trans activator protein. Cell 55: 1179-1188, 1988.

7. Elliott G and O'Hare P: Intercellular trafficking and protein delivery by a herpesvirus structural protein. Cell 88: 223-233, 1997.

8. Muthumani K, Lambert VM, Shanmugam M, Thieu KP, Choo AY, Chung JC, Satishchandran A, Kim JJ, Weiner DB and Ugen KE: Anti-tumor activity mediated by protein and peptide transduction of HIV viral protein $\mathrm{R}(\mathrm{Vpr})$. Cancer Biol Ther 8: 180-187, 2009.

9. Liu CS, Kong B, Ma DX, Wang WX, Mq W and Kao E: VP22 enhanced intercellular trafficking of HSV thymidine kinase promoted an effective cell killing effect at lower concentration of ganciclovir. Chin J Cancer Biother 8: 93-97, 2001. 
10. Albarran B, To R and Stayton PS: A TAT-streptavidin fusion protein directs uptake of biotinylated cargo into mammalian cells. Protein Eng Des Sel 18: 147-152, 2005.

11. Xiong F, Xiao S, Peng F, Zheng H, Yu M, Ruan Y, Li W, Shang Y, Zhao C, Zhou W, et al: Herpes simplex virus VP22 enhances adenovirus-mediated microdystrophin gene transfer to skeletal muscles in dystrophin-deficient (mdx) mice. Hum Gene Ther 18: 490-501, 2007.

12. Ivanenkov VV, Felici F and Menon AG: Targeted delivery of multivalent phage display vectors into mammalian cells. Biochim Biophys Acta 1448: 463-472, 1999.

13. Schwarze SR, Hruska KA and Dowdy SF: Protein transduction: Unrestricted delivery into all cells? Trends Cell Biol 10: 290-295, 2000.

14. Ho A, Schwarze SR, Mermelstein SJ, Waksman G and Dowdy SF: Synthetic protein transduction domains: Enhanced transduction potential in vitro and in vivo. Cancer Res 61: 474-477, 2001.

15. Rajotte D and Ruoslahti E: Membrane dipetidase is the receptor for a lung-targeting peptide dentified by in vivo phage display. J Biol Chem 274: 11593-11598, 1999.

16. Dente L, Vetriani C, Zucconi A, Pelicci G, Lanfrancone L, Pelicci PG and Cesareni G: Modified phage peptide libraries as a tool to study specificity of phosphorylation and recognition of tyrosine containing peptide. J Mol Biol 269: 694-703, 1997.

17. Liu F, Guo XR, Gong HX, Ni YH, Fei L, Pan XQ, Guo M and Chen RH: A resistin binding peptide selected by phage display inhibits 3T3-L1 preadipocyte differentiation. Chin Med J (Engl) 119: 496-503, 2006.

18. Pires D, Bemquerer M and Nascimento C: Some mechanistic aspects on Fmoc solid phase peptide synthesis. Int J Pept Res Ther 20: 53-69, 2014.

19. West MA, Bretscher MS and Watts C: Distinct endocytotic pathways in epidermal growth factor-stimulated human carcinoma A431 cells. J Cell Biol 109: 2731-2739, 1989.

20. Furuchi T and Anderson RG: Cholesterol depletion of caveolae causes hyperactivation of extracellular signal-related kinase (ERK). J Biol Chem 273: 21099-21104, 1998.

21. Subtil A, Hémar A and Dautry-Varsat A: Rapid endocytosis of interleukin 2 receptors when clathrin-coated pit endocytosis is inhibited. J Cell Sci 107: 3461-3468, 1994.

22. Garcia-Lora A, Algarra I and Garrido F: MHC class I antigens, immune surveillance and tumor immune escape. J Cell Physiol 195: 346-355, 2003.

23. Adessi C, Miege C, Albrieux C and Rabilloud T: Two-dimensional electrophoresis of membrane proteins: A current challenge for immobilized pH gradients. Electrophoresis 18: 127-135, 1997.

24. Huebener N, Lange B, Lemmel C, Rammensee HG, Strandsby A Wenkel J, Jikai J, Zeng Y, Gaedicke G and Lode HN: Vaccination with minigenes encoding for novel 'self' antigens are effective in DNA-vaccination against neuroblastoma. Cancer Lett 197: 211-217, 2003.

25. Siegel R, Naishadham D and Jemal A: Cancer statistics, 2013. CA Cancer J Clin 63: 11-30, 2013.
26. Early Breast Cancer Trialists' Collaborative Group (EBCTCG) Effects of chemotherapy and hormonal therapy for early breast cancer on recurrence and 15-year survival: An overview of the randomised trials. Lancet 365: 1687-1717, 2005.

27. Dean-Colomb W and Esteva FJ: Her2-positive breast cancer: Herceptin and beyond. Eur J Cancer 44: 2806-2812, 2008.

28. Davoli A, Hocevar BA and Brown TL: Progression and treatment of HER2-positive breast cancer. Cancer Chemother Pharmacol 65: 611-623, 2010.

29. Fogelman DR, Kopetz S and Eng C: Emerging drugs for colorectal cancer. Expert Opin Emerg Drugs 13: 629-642, 2008.

30. Kim R and Toge T: Changes in therapy for solid tumors: Potential for overcoming drug resistance in vivo with molecular targeting agents. Surg Today 34: 293-303, 2004.

31. Ran JT, Zhou YN, Tang CW, Lu JR, Wu J, Lu H and Yang GD: Celecoxib induces apoptosis and inhibites angiogenesis in gastric cancer. Zhonghua Zhong Liu Za Zhi 30: 448-451, 2008 (In Chinese).

32. Ortega J, Vigil CE and Chodkiewicz C: Current progress in targeted therapy for colorectal cancer. Cancer Control 17: 7-15, 2010.

33. Mailliez A, Baldini C, Van JT, Servent V, Mallet Y and Bonneterre J: Nasal septum perforation: A side effect of bevacizumab chemotherapy in breast cancer patients. Br J Cancer 103: 772-775, 2010

34. Takeda A, Loveman E, Harris P, Hartwell D and Welch K: Time to full publication of studies of anti-cancer medicines for breast cancer and the potential for publication bias: A short systematic review. Health Technol Assess 12: 1-46, 2008.

35. Brown KC: Peptidic tumor targeting agents: The road from phage display peptide selections to clinical applications. Curr Pharm Des 16: 1040-1054, 2010.

36. Laakkonen $\mathrm{P}$ and Vuorinen $\mathrm{K}$ : Homing peptides as targeted delivery vehicles. Integr Biol (Camb) 2: 326-337, 2010.

37. Miller WH, Alberts DP, Bhatnagar PK, Bondinell WE, Callahan JF, Calvo RR, Cousins RD, Erhard KF, Heerding DA, Keenan RM, et al: Discovery of orally active nonpeptide vitronectin receptor antagonists based on a 2-benzazepine gly-Asp mimetic. J Med Chem 43: 22-26, 2000.

38. Zhao H, Wang JC, Sun QS, Luo CL and Zhang Q: RGD-based strategies for improving antitumor activity of paclitaxel-loaded liposomes in nude mice xenografted with human ovarian cancer. J Drug Target 17: 10-18, 2009.

39. Dong J, Liu WQ, Jiang AM, Zhang KJ and Chen MQ: A novel peptide, selected from phage display library of random peptides, can efficiently target into human breast cancer cell. Chinese Science Bulletin 53: 860-867, 2008.

40. Zitzmann S, Krämer S, Mier W, Hebling U, Altmann A, Rother A, Berndorff D, Eisenhut $M$ and Haberkorn U: Identification and evaluation of a new tumor cell-binding peptide, FROP-1. J Nucl Med 48: 965-972, 2007. 